\title{
The formation of multiple layers of ice particles in the polar summer mesopause region
}

\author{
H. Li ${ }^{1,2}$, J. $\mathbf{W u}^{2}$, and Z. Zhou ${ }^{1}$ \\ ${ }^{1}$ Department of physics, Harbin Institute of Technology, Harbin, China \\ ${ }^{2}$ China Research Institute of Radio wave Propagation (CRIRP), Beijing, China \\ Correspondence to: H. Li (lihui_2253@163.com)
}

Received: 19 October 2015 - Revised: 11 January 2016 - Accepted: 13 January 2016 - Published: 25 January 2016

\begin{abstract}
This paper presents a two-dimensional theoretical model to study the formation process of multiple layers of small ice particles in the polar summer mesosphere as measured by rockets and associated with polar mesosphere summer echoes (PMSE). The proposed mechanism primarily takes into account the transport processes induced by gravity waves through collision coupling between the neutral atmosphere and the ice particles. Numerical solutions of the model indicate that the dynamic influence of wind variation induced by gravity waves can make a significant contribution to the vertical and horizontal transport of ice particles and ultimately transform them into thin multiple layers. Additionally, the pattern of the multiple layers at least partially depends on the vertical wavelength of the gravity wave, the ice particle size and the wind velocity. The results presented in this paper will be helpful to better understand the occurrence of multiple layers of PMSE as well as its variation process.
\end{abstract}

Keywords. Ionosphere (ionospheric irregularities)

\section{Introduction}

The summer polar mesosphere is one of the most intriguing regions of the Earth's upper atmosphere for scientific study. It exhibits a rich variety of geophysical phenomena that are attributable to its unique thermal structure and complicated dynamic processes. Noctilucent clouds (NLCs) and polar mesospheric summer echoes (PMSE) are two phenomena which have been intensely investigated, both experimentally and theoretically, over the last 2 decades (Ecklund and Balsley, 1981; Röttger et al., 1988; Hocking and Röttger, 1997). NLCs are formed primarily from larger ice particles, which have been observed by the naked eye throughout his- tory (Gadsden et al., 1998). PMSE are strong radar backscatters that have been observed at frequencies from $50 \mathrm{MHz}$ to $1.3 \mathrm{GHz}$. Several theories have been put forward to explain the PMSE since they were first detected by the Poker Flat $50 \mathrm{MHz}$ radar in 1979. Although it is known that charged ice particles in the mesosphere likely play a crucial role in creating the PMSE (Reid, 1990; Havnes et al., 1992; Cho et al., 1997; Lie-Svendsen et al., 2003; La Hoz et al., 2006), several questions remain unanswered. The review by Rapp and Lübken (2004) provides detailed information about the characteristics of PMSE and their relation to other mesosphere phenomena.

In order to investigate the mechanism of the PMSE phenomena, studies on the relationship between PMSE and ice particle behavior has been conducted using ground-based radars and in situ rocket sounding when the PMSE happen. A remarkable feature of all measuring results is the fact that the ice particles often occur in the form of distinct layered structures and the strong echoes from the altitude range often correspond to these layer regions (Ulwick et al., 1988; Havnes et al., 2001). Although there are numerous experimental reports about these multiple layers, less theoretical work has been put forward to explain their origin as well as formation process. Currently, the underlying physical processes leading to these multiple layer structures are not well understood.

Since the ice particle formation strongly depends on the extremely cold temperature of the mesopause, some authors proposed that a gravity wave causes the temperature variation, which leads to the growth and concentration of ice particles in a cold phase (Klostermeyer, 1997). Based on this idea, it has been suggested that a correlation exists between the ice layers and the vertical gradient of the temperature structure. Such a suggestion, however, has been questioned because the 
thermal structure based on techniques using rocket measurements reveals that there are no distinct multiple layer temperature structures located in the mesopause region (Lübken, 1999). Additionally, the temperature minimum is repeatedly observed at approximately $87 \mathrm{~km}$, whereas the altitude of the ice layers is frequently observed below this elevation. Thus, this hypothesis still requires rigorous testing.

The spatial structure or time duration from radar echoes often present a wavy pattern, due to an effect that is attributed to their interaction with gravity waves. Accordingly, numerous studies have been carried out to analyze a possible connection between ice particle layers and gravity waves (Hoppe et al., 1998; Rüster et al., 1996). Recently, the relationship between the PMSE and gravity waves was further supported by the results of a study by Hoffmann et al. (2008), who showed the strongest correlation between the PMSE with a double layer structure and gravity waves for periods of 5-7 h based on their continuous MF radar wind measurement. All these investigations imply that gravity waves were the dominant contributor to the formation of multiple layers of ice particle. However, even though it is generally believed that a close correlation exists between gravity waves and multiple layers, several previous studies considered the gravity waveinduced temperature fluctuations on ice chemistry or other microphysical processes, but none considers the effects of gravity wave dynamics on this process, and a rational theoretical model conforming to observations has yet to be put forward.

The specific purpose of this paper is to present a mechanism that produces multiple layers of ice particles in the polar summer mesosphere region. The hypothesis is that the transport of heavy ice particles associated with gravity waveinduced winds causes a variation in the density of ice particles, through collision coupling between the neutral atmosphere and the ice particle, and ultimately might cause their convergence into thin multiple layers. This physical process has been described in detail in our previous simplified onedimensional model ( $\mathrm{Li}$ et al., 2010). In this paper, we have addressed these processes by using a time-dependent twodimensional model. The model and potential physical mechanisms are described in Sect. 2, the results are presented in Sect. 3 and the discussion and summary are presented in Sect. 4.

\section{Theoretical model}

We propose to make an assessment of whether the motion associated with gravity waves can lead to the formation of multiple layers of ice particles. The density variations of ice particles are described by the continuity equation.

$$
\frac{\partial n_{\mathrm{d}}}{\partial t}+\nabla \cdot\left(n_{\mathrm{d}} u_{d}\right)=0
$$

where $\nabla=\hat{x}(\partial / \partial x)+\hat{y}(\partial / \partial y)$ and $n_{\mathrm{d}}$ and $u_{d}$ are the ice particle density and velocity, respectively. Several assumptions are made to obtain the solutions presented in this paper. It is assumed the ice particle size and total density reaches a steady state. This implies that all particle microphysics including ice growth, sublimation and sedimentation effects are not considered. In addition, if we impose gravity wave perturbations at this point, any changes in layers properties could be attributed to the wave winds. Therefore, our current considerations will be regarded as indicative rather than quantitative results. Strictly speaking, the forces acting on the ice particles include the drag force, atmospheric pressure and gravity in the mesopause region. The motion velocity of the ice particle is given by the momentum equation as

$m_{d} n_{\mathrm{d}} \frac{\partial u_{d}}{\partial t}=-n_{\mathrm{d}} m_{d} v_{\mathrm{d} n}\left(u_{d}-u_{\mathrm{n}}\right)-\frac{\partial p_{d}}{\partial z}+m_{d} n_{\mathrm{d}} g$.

Here $m_{d}$ is the mass of the ice particle and $v_{\mathrm{d} n}$ is the effective collision frequency. $p_{d}=n_{\mathrm{d}} k T_{d}$ is the partial pressure and $g$ is the gravitational acceleration. The effective collision frequency $v_{d n}$ for the ice particles is represented by (LieSvenson et al., 2003):

$v_{\mathrm{d} n}=\frac{8}{3 \sqrt{\pi}} \frac{n_{\mathrm{n}} m_{\mathrm{n}}}{m_{d}+m_{\mathrm{n}}} \sqrt{\frac{2 k_{B} T\left(m_{d}+m_{\mathrm{n}}\right)}{m_{d} m_{\mathrm{n}}}} \pi\left(r_{d}+r_{n}\right)^{2}$,

where, $n_{\mathrm{n}}, m_{\mathrm{n}}$ and $r_{d}$ are the neutral density, mass and particle radius, respectively. The term $u_{\mathrm{n}}=u_{0}+v$ in Eq. (2) is the velocity of the neutral particles. In the present consideration, $u_{0}=\left(u_{0}, w_{0}\right)$ is the background neutral wind velocity, $v=v\left(u^{\prime}, w^{\prime}\right)$ is assumed to represent the gravity wave wavelike perturbation velocity and $u^{\prime}$ and $w^{\prime}$ are its components of perturbation winds due to the gravity wave in the horizontal and vertical directions, respectively. The conservation equations for mass, momentum and energy govern motion in the atmosphere as follows:

$$
\begin{aligned}
& \frac{\partial \rho}{\partial t}+\frac{\partial(\rho u)}{\partial x}+\frac{\partial(\rho w)}{\partial z}=0 \\
& \frac{\partial u}{\partial t}+u \frac{\partial u}{\partial x}+w \frac{\partial u}{\partial z}+\frac{1}{\rho} \frac{\partial p}{\partial x}=0 \\
& \frac{\partial w}{\partial t}+u \frac{\partial w}{\partial x}+w \frac{\partial w}{\partial z}+\frac{1}{\rho} \frac{\partial p}{\partial z}+g=0 \\
& \frac{\partial T}{\partial t}+u \frac{\partial T}{\partial x}+w \frac{\partial T}{\partial z}+T(\gamma-1)\left(\frac{\partial u}{\partial x}+\frac{\partial w}{\partial z}\right)=0 \\
& p=\rho R T
\end{aligned}
$$

Equation (4) is used to describe the gravity wave propagation model. Where $u$ and $w$ are the horizontal and vertical components of total wind velocities, respectively; $\rho, p$ and $T$ are density, pressure and temperature, respectively; $R$ is the universal gas constant and $\gamma$ is the ratio of the specific heats.

This set of Eqs. (1-4) describes the ice particle dynamic transport process coupling with gravity waves. Numerical methods are used to solve these equations and to simulate ice particle layer formation and evolution. Additionally, the effects of the gravity wave on the layering process are considered for a range of assumed wave parameters. 


\section{The numerical method}

In order to solve Eq. (4), a numerical scheme should be of high accuracy and stability. The full-implicit continuous Eulerian scheme (FICE) has been extensively applied in numerical simulations of the gravity wave. The FICE algorithm has been previously described in detail by ( $\mathrm{Hu}$ and $\mathrm{Wu}, 1984$; Zhang and Yi, 1999). The solution methods used here are similar to the earlier simulation. The Crank-Nicholson temporal difference scheme and center spatial difference scheme were used to solve the continuity and momentum equation of the ice particle.

In general, the numerical solutions of the above equations depend upon the boundary conditions. Because the wave perturbations at each boundary are equal, we used periodic boundary conditions in the horizontal direction. Additionally, the zero flux condition is used for the velocities and temperature on the upper boundary in the vertical direction, while the density and pressure are approximated in the hydrostatic equilibrium and state equation. The lower boundary condition is kept invariable until the interior point difference equations are solved by iteration and a small Rayleigh friction is applied to avoid the boundary reflection.

The issue addressed with our theoretical model is the effect that gravity waves have on the layering of ice particles, which results in the formation of multiple layers in the PMSE region. To answer this, we first assumed the ice particle size and the initial height distribution in existence in this region. Then, we studied the density variation of the ice particles relative to the gravity wave perturbation. Since we only considered the gravity wave wind perturbations in this study, some microphysical processes were neglected and any changes in height distribution could be attributed to the gravity wave wind effects. As a reference case, we assume that after a few days the ice size and density reach a steady state and the initial vertical density distribution can be simply regarded as a Gaussian distribution.

$n_{\mathrm{d}}=n_{d 0} \exp \left(-\left(h-h_{0}\right)^{2} / 2 \sigma^{2}\right)$

The parameters assumed in this simulation were as consistent with the experimental measurements as possible. Here, $\sigma=2.5 \mathrm{~km}$ is the width of the pre-existing layers and the central height $h_{0}=86 \mathrm{~km}$.

In order to obtain the numerical solution of the model, the initial perturbation must be introduced. The initial perturbation model used here is the same as that described by Xu and Smith (2004). The initial horizontal perturbation is a Gaussian gravity wave packet with the following form:

$$
\begin{aligned}
u^{\prime}(x, z, 0)= & u_{c} \sin \left(k_{x} x+k_{z}\left(z-z_{0}\right)\right) \\
& \exp \left(-\ln 2\left(\frac{z-z_{0}}{\lambda_{z}}\right)^{2}\right) \cdot \exp \left(\frac{z-z_{0}}{2 H}\right),
\end{aligned}
$$

where $k_{x}=2 \pi / \lambda_{x}$ and $k_{z}=2 \pi / \lambda_{z}$ are the wave numbers in the horizontal and vertical directions respectively and $H$ is the scale height. The other initial perturbation quantities (i.e. $\left.w^{\prime}(x, z, 0), T^{\prime}(x, z, 0), \rho^{\prime}(x, z, 0)\right)$ are derived from the $u^{\prime}(x, z, 0)$ using the polarization equations of the gravity waves in the linear case (Fritts and Alexander, 2003).

\section{Results of the simulation}

\subsection{The solution of a reference case}

The rocket data revealed a wide range of vertical wavelength (3-7 km) near the mesopause. A typical horizontal wavelength is around $100-1000 \mathrm{~km}$. For a reference case, the values of the horizontal wavelength and the vertical wavelength were chosen to be 200 and $4 \mathrm{~km}$, respectively. The other parameters in the model calculation are the neutral atmosphere number density $n_{\mathrm{n}}=1 \times 10^{20} \mathrm{~m}^{3}$, the particle radius $r_{d}=10 \mathrm{~nm}$, and $m_{d} / m_{\mathrm{n}} \approx 10^{5}$. In the horizontal direction, the calculation domain is one wavelength and the vertical domain extends from the 78 to $92 \mathrm{~km}$.

A time series of numerical results illustrating the ice particle layering process when the gravity wave propagates through the mesopause region is shown in Fig. 1. The layers are displayed as a function of the horizontal distance and height, and the time interval is half an hour for each panel after the gravity wave perturbations were initiated. The results indicate that a single thick charged ice layer can transform into distinct thin multi-layer structures as a result of the gravity wave perturbation. Some detailed features are outlined below. After the model runs for half an hour (Fig. 1b), the wave induces an undulation and distorts the pre-existing layers (Fig. 1a). When we let the model run for $2.5 \mathrm{~h}$, two distinct layers appear, located at approximately 84 and $88 \mathrm{~km}$, respectively, and the thickness of each layer is equal. Notably, the ice layers can continuously vary with the cyclical activity of the gravity. In addition, given that the diffusion of the ice particles is very slow, the layered structure of the ice particles can be maintained for a long period of time when the gravity waves disappear. These results are consistent with observations. The other important feature illustrated by our simulation is that the trend of the layers is gradually downward, which is due to the phase velocity of the gravity wave being directed downward. Actually, this downward moving reflecting layer is frequently observed in many PMSE experiments.

\subsection{The effect of the gravity wave parameters}

According to our model and the results of the above simulation, the pattern of multi-layer structure formation and evolution primarily depends upon the gravity wave parameters (including amplitude, wavelength, period, etc.) and the pre-existing density distribution. For example, the sounding rocket measurements show that the ice particles appear often in one, two or even more layers. To explain the origin of these layering phenomena, based on the above hypothesis of grav- 

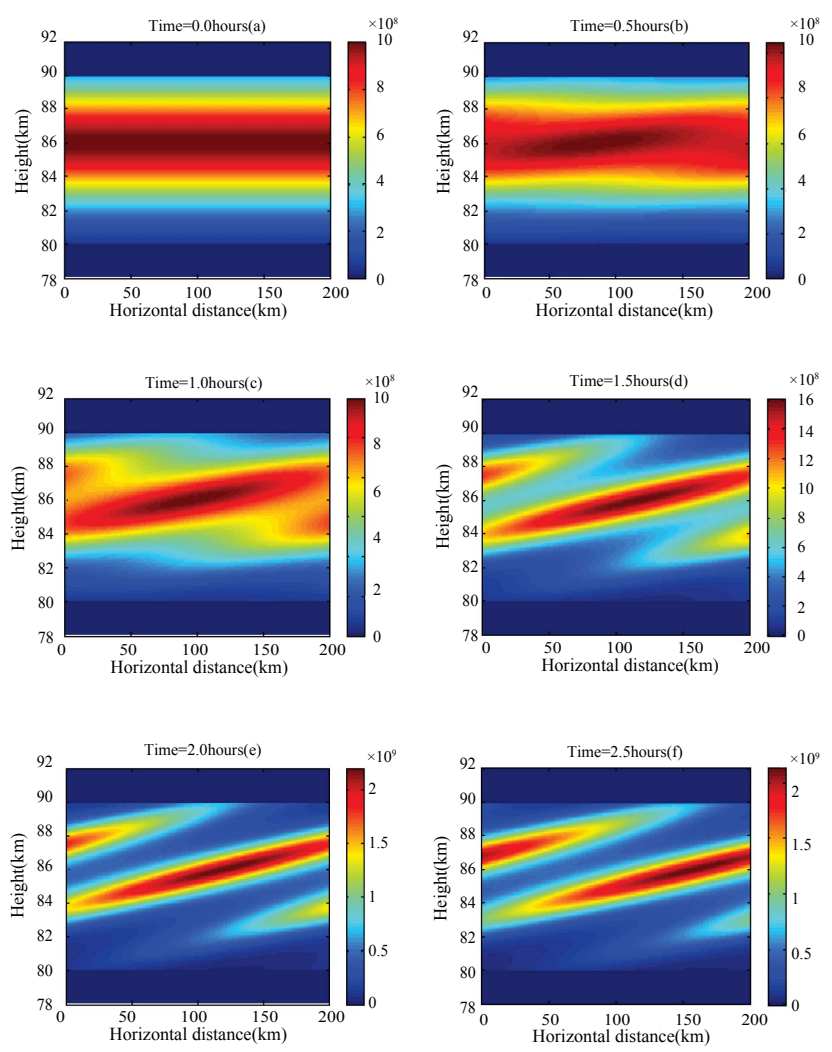

Figure 1. Ice particle layer formation and evolution plotted versus horizontal distance and height for gravity wave perturbations. The time interval is half an hour $(t=0.0,0.5,1.0,1.5,2.0,2.5 \mathrm{~h})$ for each panel.

ity wave perturbation, we examine the effect of the vertical wavelength on the formation of multiple layers. The structure of ice particle layers with different vertical wavelengths, namely $\lambda=3,4$ and $5 \mathrm{~km}$, respectively, is shown in Fig. 2 . The other parameters were the same as in Fig. 1. Additionally, as a reference case and in order to see more clearly, we only show one-dimensional results, and the vertical profiles of ice layers for a fixed horizontal position of $x=100 \mathrm{~km}$ were calculated when the model was run for 3 hours. In addition, as illustrated in Fig. 2, when $\lambda=3 \mathrm{~km}$, three layers are produced, when $\lambda=4 \mathrm{~km}$ two layers are produced and when $\lambda=5 \mathrm{~km}$ only one obvious single layer is observed. Moreover, as seen in Fig. 2, as the number of layers decreases, the vertical thickness of the layers increases. These features are consistent with multiple experimental observations by sounding rockets. Besides the vertical wavelength, other gravity wave parameters also affect the formation and the pattern of multiple layers. For instance, the bigger the perturbation velocity, the faster and easier it is for ice particles to form the layered structure.

\subsection{Effect of ice particle size}

In the above results, we assumed that the ice particles have a uniform radius $r_{d}=10 \mathrm{~nm}$, but the descent speed of a larger particle would be higher than that of a smaller particle of the same density. Therefore, layer formation and evolution with different particle sizes is also considered in this study. The reference case for three different sizes of ice particle, namely $r_{d}=10,20$ and $30 \mathrm{~nm}$, respectively, while the other parameters are held the same as in Fig. 1, is presented in Fig. 3, where it can be seen that the particle size also influences the formation of layers. Meanwhile, when the gravity wave parameters were fixed, the altitude of the layers decreases more quickly and their formation become more difficult with increasing ice particle size. Furthermore, the smaller the particle is, the easier the formation of layered structures and the shift or disappearance is faster than in the case of the larger particle size. Therefore, based on the results in Figs. 2 and 3, we feel confident that the pattern of the multi-layer structure, at least partially, depends on the vertical wavelength of the gravity wave, the ice particle size and the wind velocity caused by the gravity wave.

\subsection{Experimental case study}

To confirm the theoretical approach outlined in this paper, we compared our model calculation results with the rocket measurements. Figure 4 shows the experimental data of the temperature profile (left) and the charged ice particle density distribution (right, thin black line) collected during the ECT02 rocket sounding campaign. The results depicted in this figure reveal that the charged ice particles occur in distinct double layers during this experiment, and these two layers are located at an altitude of approximately 85.6 and $88 \mathrm{~km}$, respectively. Also, as noted earlier, there is no distinctly layered structure in the corresponding temperature profile. Moreover, the results show that the two layers have almost the same width, while the temperature differential between these two layers is approximately $10 \mathrm{~K}$. Clearly, temperature is not the main factor for the formation of such layered structures.

To understand the layering process described above, we assumed the initial ice particle distribution in our model calculation. The initial distribution is a Gaussian distribution with the central height at $h=87.5 \mathrm{~km}$ (thin blue line), which corresponds to the minimum temperature during this experiment (Fig. 4, left panel). Without being able to measure the gravity wave parameters during the ECT02 campaign, we can only estimate the gravity wave parameters based on the experimental data to simulate the layer formation. For example, we input the vertical wavelength of gravity waves as $2.5 \mathrm{~km}$ in the model calculation. This numerical value is derived based on the distance between the two layers. The other parameters are the same as in Fig. 2, the model calculation result (red line). 

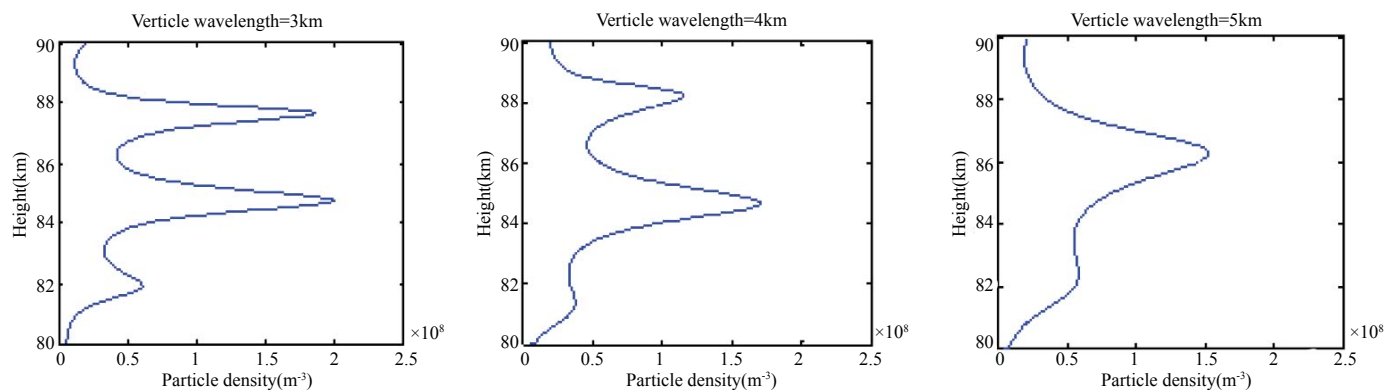

Figure 2. The effect of vertical wavelength on the formation of ice particle layers, the wavelength $\lambda=3,4$ and $5 \mathrm{~km}$, respectively.

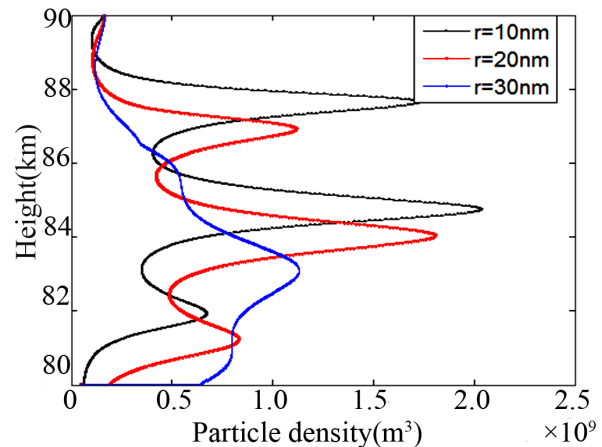

Figure 3. The formation of layered structures with different ice particle sizes Radius $r_{d}=10,20,30 \mathrm{~nm}$.
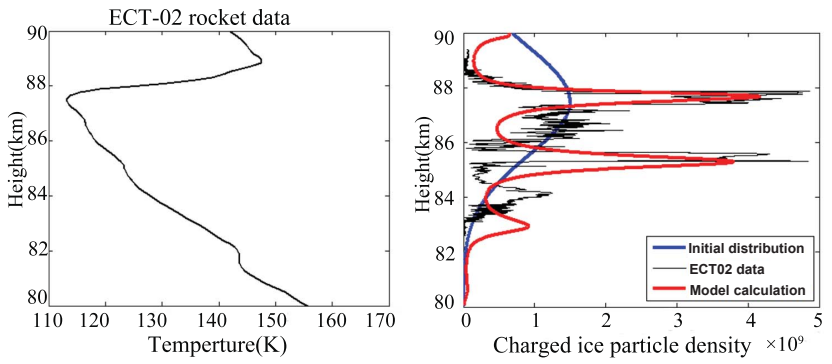

Figure 4. The vertical profile of the temperature (left) and charged ice density distribution (right) from the ECT02 rocket sounding (black line). The initial distribution of charged ice particles (blue line) and the model calculation results (red line).

The result presented in Fig. 4 indicates that the theoretical result is consistent with the experimental measurements when reasonable gravity wave parameters are chosen. However, while this simulation result can explain the observed results, any quantitative simulation requires complete knowledge of these parameters within the layers throughout the entire period of their formation.

\section{Discussion and conclusion}

In this paper, we have developed a two-dimensional theoretical model to examine the effects of gravity waves on the formation of multiple layers of ice particle in the polar summer mesosphere. Although certain previous explanations suggested the involvement of gravity wave, one aspect that has not previously been considered is the transport process caused by gravity wave-induced winds in the horizontal and vertical directions. These perturbation winds cause regions of ice particle convergence and divergence and ultimately result in the formation of layers. To describe this physical process, numerical simulations are used to show how a preexisting thick, single ice layer transformed into thin multiple layers when gravity waves propagate through the mesopause region. The results also indicate that the pattern of the multilayer structure depends on the vertical wavelength of the gravity waves, the ice particle size and the wind velocity. This mechanism can explain most types of observations.

To distinguish between the different effects on the layering processes, our model included only the dynamic effects caused by the gravity wave wind perturbations. Additionally, particle microphysics was not considered, and as such, the ice size and its density remain constant in our model. According to the kinetic theory approximation model (Reid, 1975), the rate of growth of the radius of a spherical ice particle is approximately $0.06 \mathrm{~nm}$ per minute, thus ice particles with a radius between 5 and $20 \mathrm{~nm}$ require 6 hours or more to develop. Accordingly, the ice particle size can affect the process of layer formation. However, the growth process is minor compared with the transport process caused by gravity waves, and particle size was not given a detailed consideration in this study.

Importantly, in the current study we have primarily considered possible layering mechanisms leading to ice particles forming multiple layers. The relationship between the ice layers and strong radar echoes has not been addressed here. It is assumed that the interaction of these ice particles with the ambient plasma can create the structure in the medium necessary for the scattering of the radio waves. Reid (1990) has shown how small ice particles can acquire a charge by scavenging the free electrons. This charging not only forms 
the gradients at the boundaries of the depletion regions, but also increases their lifetime by reducing the electron diffusion through the Schmidt number effect. So we feel confident that the layering process presented in this paper will be helpful for a better understanding of the generation mechanism of the PMSE, as well as the variation of some of their properties, such as the multiple PMSE layers.

To further confirm the ideas presented in this paper, our current understanding of the mechanism will undoubtedly require more extensive in situ and ground-based observations in the future. For example, simultaneous monitoring of the gravity waves and ice particle layers during PMSE observations in the polar mesopause.

Acknowledgements. The authors wish to express his sincere thanks to Bin-Xu for his some constructive comments. Our appreciate O. Havnes, University of Troms $\varnothing$, Norway, for providing ECT02 data. This work was supported by the National Science Foundation of China (NSFC) under grant 40831062.

The topical editor, A. J. Kavanagh, thanks X.-H. Xue and M. Kosch for help in evaluating this paper.

\section{References}

Cho, J. Y. N. and Röttger, J.: An updataed review of polar mesosphere summer ehcoes: observation, theory, and their relationship to noctilucent clouds and subvisible aerosols, J. Geophys. Res., 102, 2001-2020, 1997.

Ecklund, W. L. and Balsley, B. B.: Long-term observations of the arctic mesosphere with the MST radar at Poker Flat, Alaska, J. Geophys. Res., 86, 7775-7780, 1981.

Fritts, D. C. and Alexander, M. J.: Gravity wave dynamics and effects in the middle atmosphere, Rev. Geophys., 41, 1003, doi:10.1029/2001RG000106, 2003.

Gadsden, M.: The north-west Europe data on noctilucent clouds: A survey, J. Atmos. Sol Terr-Phy., 60, 1163-1174, 1998.

Havnes, O., Melandsø, F., Hoz, C. L., Aslaksen, T. K., and Hartquist, T.: Charged dust in the Earth's mesopause: Effects on radar backscatter, Phys. Scripta, 45, 535-544, 1992.

Havnes, O., Trøim, J., Blix, T., Mortensen, W., Naesheim, L. I., Thrane, E., and Tønnesen, T.: First detection of charged dusty particles in the earth's mesosphere, J. Geophys. Res., 101, 10839-10847, 1996.

Havnes, O., Brattli, A., Aslaksen, T., Singer, W., Latteck, R., Blix, T., Thrane, E., and Trøim, J.: First common volume observations of layered plasma structures and polar mesospheric summer echoes by rocket and radar, Geophys. Res. Lett., 28, 1419-1422, 2001.

Hocking, W. K. and Röttger, J.: Studies of polar mesosphere summer echoes over EISCAT using calibrated signal strengths and statistical parameters, Radio Sci., 32, 1425-1444, 1997.
Hoffmann, P., Rapp, M., Fiedler, J., and Latteck, R.: Influence of tides and gravity waves on layering processes in the polar summer mesopause region, Ann. Geophys., 26, 4013-4022, doi:10.5194/angeo-26-4013-2008, 2008.

Hoppe, U.-P., Hall, C., and Röttger, J.: First observations of summer polar mesospheric backscatter with a $224 \mathrm{MHz}$ radar, Geophys. Res. Lett., 15, 28-31, 1998.

$\mathrm{Hu}$, Y. Q. and Wu, S. T.: A full-implicit-Continous-Eulerian (FICE) scheme for multidimensional transient magneto dynamic flows, J. Comput. Phys., 55, 33-64, 1984.

Klostermeyer, J.: A height and time-dependent model of polar mesosphere summer echoes, J. Geophys. Res., 102, 6715-6727, 1997.

La Hoz, C., Havnes, O., Naesheim, L. I., and Hysell, D. L.: Observations and theories of polar mesospheric summer echoes at a Bragg wavelength of $16 \mathrm{~cm}$, J. Geophys. Res., 111, D04203, doi:10.1029/2005JD006044, 2006.

Li, H., Wu, J., Wu, J., Xu, Z.-W., and Xu, B.: Study on the layered dusty plasma structures in the summer polar mesopause, Ann. Geophys., 28, 1679-1686, doi:10.5194/angeo-28-16792010, 2010.

Lie-Svendsen, O., Blix, T. A., Hoppe, U., and Thrane, E.: Modelling the plasma response to small-scale particle perturbations in the mesopause region, J. Geophys. Res., 108, 8442, doi:10.1029/2002JD002753, 2003.

Lübken, F.-J.: Thermal structure of the arctic summer mesosphere, J. Geophys. Res., 104, 9135-9149, 1999.

Rapp, M. and Lübken, F.-J.: Polar mesosphere summer echoes (PMSE): Review of observations and current understanding, Atmos. Chem. Phys., 4, 2601-2633, doi:10.5194/acp-4-2601-2004, 2004.

Reid, G. C.: Ice clouds at the summer polar mesopause, J. Atmos. Sci, 32, 523-535, 1975.

Reid, G. C.: Ice particles and electron "bite-outs" at the summer polar mesopause, J. Geophys. Res., 95, 13891-13896, 1990.

Röttger, J., Hoz, C. L., Kelly, M. C., Hoppe, U.-P., and Hall, C.: The structure and dynamics of polar mesosphere summer echoes observed with the EISCAT $224 \mathrm{MHz}$ radar, Geophys. Res. Lett., 15, 1353-1356, 1988.

Rüster, R., Czechnowsky, P., Hoffmann, P., and Singer, W.: Gravity wave signatures at mesopause heights, Ann. Geophys, 14, 11861191, 1996.

Ulwick, J. C., Baker, K. D., Kelley, M. C., Balsley, B. B., and Ecklund, W. L.: Comparison of simultaneous MST radar and electron density probe measurements during STATE, J. Geophys. Res., 93, 6989-7000, 1988.

$\mathrm{Xu}$, J. and Smith, A. K.: Studies of gravity wave induced fluctuations of the sodium layer using linear and nonlinear models, J. Geophys. Res., 109, D02306, doi:10.1029/2003JD004038, 2004.

Zhang, S. D. and Yi, F.: A numerical study of nonlinear propagation of a gravity wave packet in compressible atmosphere, J. Geophys. Res., 104, 14261-14270, 1999. 